\title{
An Engel condition with an additive mapping in semiprime rings
}

\author{
MAJA FOŠNER ${ }^{1}$, NADEEM UR REHMAN ${ }^{2}$ \\ and JOSO VUKMAN ${ }^{3}$
}

${ }^{1}$ Faculty of Logistics, University of Maribor, Mariborska cesta 7, 3000 Celje, Slovenia
2Department of Mathematics, Aligarh Muslim University, Aligarh 202 002, India
${ }^{3}$ Department of Mathematics, Physics and Mechanics, University of Maribor,
Gosposvetska 84, 2000 Maribor, Slovenia
E-mail: maja.fosner@ @uni-mb.si; rehman100@gmail.com; joso.vukman@ uni-mb.si

MS received 16 July 2013; revised 19 October 2013

\begin{abstract}
The main purpose of this paper is to prove the following result: Let $n>1$ be a fixed integer, let $R$ be a $n$ !-torsion free semiprime ring, and let $f: R \rightarrow R$ be an additive mapping satisfying the relation $[f(x), x]_{n}=[[\ldots[[f(x), x], x], \ldots], x]=0$ for all $x \in R$. In this case [ $f(x), x]=0$ is fulfilled for all $x \in R$. Since any semisimple Banach algebra (for example, $C^{*}$ algebra) is semiprime, this purely algebraic result might be of some interest from functional analysis point of view.
\end{abstract}

Keywords. Prime ring; semiprime ring; additive mapping; derivation; commuting mapping; centralizing mapping; functional identity.

2010 Mathematics Subject Classification. 16N60, 16R50.

Throughout, $R$ will represent an associative ring with a centre $Z(R)$. A ring $R$ is $n$-torsion free, where $n>1$ is an integer, in case $n x=0, x \in R$, implies $x=0$. As usual the commutator $x y-y x$ will be denoted by $[x, y]$. Set $[y, x]_{1}=[y, x]$ for any $x, y \in R$, and for $n>1$, let $[y, x]_{n}=\left[[y, x]_{n-1}, x\right]$. Recall that a ring $R$ is prime if for $a, b \in R$, $a R b=\{0\}$ implies that either $a=0$ or $b=0$, and is semiprime if $a R a=\{0\}$ implies $a=0$. We denote by $C, Q$ and $R C \subseteq Q$ the extended centroid, the maximal right ring of quotients and a central closure of a semiprime ring $R$, respectively. For the explanation of $C, Q$ and $R C$ we refer the reader to [3]. We denote by $\operatorname{char}(R)$ the characteristic of a prime ring $R$. An additive mapping $D$ of a ring $R$ into itself is called a derivation if $D(x y)=D(x) y+x D(y)$ holds for all pairs $x, y \in R$. A mapping $f$ of a ring $R$ into itself is called centralizing on $R$ if $[f(x), x] \in Z(R)$ holds for all $x \in R$. In the special case, when $[f(x), x]=0$ holds for all $x \in R$ the mapping $f$ is said to be commuting on $R$. A classical result of Posner (Posner's second theorem) [12] states that the existence of a nonzero centralizing derivation on a prime ring forces the ring to be commutative. Posner's second theorem in general cannot be proved for semiprime rings as the following example shows. Take prime rings $R_{1}, R_{2}$, where $R_{1}$ is commutative, and set $R=R_{1} \oplus R_{2}$. Let $D_{1}: R_{1} \rightarrow R_{1}$ be a nonzero derivation. A mapping $D: R \rightarrow R$, defined by $D\left(\left(r_{1}, r_{2}\right)\right)=\left(D_{1}\left(r_{1}\right), 0\right)$ is then a nonzero commuting derivation. It is not difficult to show that if $D: R \rightarrow R$ is a commuting derivation on a semiprime ring $R$, then $D$ maps $R$ into $Z(R)$. 
It is our aim in this paper to prove the following result.

Theorem 1. Let $n>1$ be a fixed integer, let $R$ be a $n$ !-torsion free semiprime ring, and let $f: R \rightarrow R$ be an additive mapping satisfying the relation

$$
[f(x), x]_{n}=0
$$

for all $x \in R$. In this case, $f$ is commuting on $R$.

In case $n=2$, the above result reduces to Theorem 4 of [16]. Since any semisimple Banach algebra is semiprime (for example, $C^{*}$ algebra), Theorem 1 might be of some interest from functional analysis point of view.

Let us see in some more details the background and the motivation of Theorem 1. Vukman [13] proved extensions of Posner's second theorem. More precisely, he proved the following result: Let $R$ be a prime ring with $\operatorname{char}(R) \neq 2$. Suppose there exists a nonzero derivation $D: R \rightarrow R$, such that the mapping $x \mapsto[D(x), x]$ is commuting on $R$. In this case $R$ is commutative. In [14], Vukman proved the following result: Let $R$ be a prime ring with $\operatorname{char}(R) \neq 2,3,5$. Suppose there exists a nonzero derivation $D: R \rightarrow R$, such that the mapping $x \mapsto[[D(x), x], x]$ is centralizing on $R$. In this case, $R$ is commutative (see also [15]).

Using the theory of differential identities, Lanski [11] fairly generalizes the results we have just mentioned by proving the following result: Let $I$ be a nonzero ideal of a prime ring $R$, and let $D: R \rightarrow R$ be a nonzero derivation, such that $[D(x), x]_{n}=0$ holds for all $x \in I$ and some fixed integer $n \geq 1$. In this case, $R$ is commutative. In the same paper, Lanski proved the result which tells: Let $R$ be a prime ring, $L$ a noncommutative Lie ideal of $R$ and $D: R \rightarrow R$ a nonzero derivation. If $[D(x), x]_{n}=0$ holds for all $x \in L$ and some fixed integer $n \geq 1$, then $\operatorname{char}(R)=2$ and $R \subseteq M_{2}(\mathbb{F})$ for $\mathbb{F}$ a field, so $[D(x), x]_{2}=0$. Brešar [5] has proved that in case an additive mapping $f: R \rightarrow R$, where $R$ is a noncommutative prime ring, is commuting on $R$, then $f$ is of the form $f(x)=\lambda x+\zeta(x)$, where $\lambda \in C$ is some fixed element, and $\zeta: R \rightarrow C$ is an additive mapping. With this result, the development of the theory of functional identities (BrešarBeidar-Chebotar theory) started. We refer the reader to [7] for an introductory account of the Brešar-Beidar-Chebotar theory. For full treatment of this sophisticated and powerful theory, we refer the reader to [8]. In [4], Brešar proved that in case we have an additive mapping $f: R \rightarrow R$, where $R$ is a prime ring with $\operatorname{char}(R) \neq 2$, such that $[f(x), x]_{2}=0$ for all $x \in R$, then $f$ is commuting on $R$. This result has been generalized to 2-torsion free semiprime rings in [16]. Using the theory of functional identities Brešar [6] has proved the following theorem: Let $R$ be a prime ring and let $f: R \rightarrow R$ be an additive mapping. Suppose there is a fixed integer $n>1$ such that $[f(x), x]_{n}=0$ holds for all $x \in R$. If $\operatorname{char}(R)=0$ or $\operatorname{char}(R)>n$, then $f$ is commuting on $R$.

The work of Beidar et al. [2] should be mentioned. They studied an additive mapping $f: I \rightarrow A$ satisfying the relation

$$
\left[\left[\ldots\left[\left[f(x), x^{n_{1}}\right], x^{n_{2}}\right], \ldots\right], x^{n_{k}}\right]=0
$$

for some fixed positive integers $n_{1}, n_{2}, \ldots, n_{k}$, and all $x \in I$, where $I$ is a right ideal of a prime ring $R$ and $A=R C$ is a central closure of $R$. They showed that in case 
either $\operatorname{char}(R)=0$ or $\operatorname{char}(R)>n_{1}+n_{2}+\cdots+n_{k}$ a mapping $f$ is commuting on $R$.

Proof of Theorem 1. As observed above, we may assume that $n>2$. In the light of comments referring to [16] we shall begin the proof. According to the semiprimeness of $R$ there exists a family of prime ideals $\left\{P_{\alpha} \mid \alpha \in I\right\}$ such that $\cap_{\alpha} P_{\alpha}=\{0\}$. Without loss of generality, we may assume that for prime rings $R_{\alpha}=R / P_{\alpha}, \operatorname{char}\left(R_{\alpha}\right)>n$ (see page 459 of [1]). By $C$ we shall denote the extended centroid of the prime ring $R / P$, for $P=P_{\alpha}$ for some $\alpha$, and by $A$ the central closure of $R / P$. One can consider $A$ as a vector space over the field $C$, which can be regarded as a subspace of $A$. Thus there exists a subspace $B$ of $A$ such that $A=B+C$. We shall denote by $\pi$ the canonical projection of $A$ onto $B$. Let us fix some $P=P_{\alpha}, \alpha \in I$. We will show that $[f(x), x] \in P$ for all $x \in R$. For $x \in R$, we shall write $\bar{x}$ for the coset $x+P \in R / P$. Write $x+p, x \in R, p \in P$, instead of $x$ in (1). It follows that $[f(p), x]_{n} \in P$ for all $x \in R$ and $p \in P$. Therefore $[\overline{f(p)}, \bar{x}]_{n}=0$ for all $x \in R$. In particular,

$$
[[\overline{f(p)}, \bar{x}], \bar{x}]_{n-1}=0 \text {. }
$$

Define a mapping $D: R / P \rightarrow R / P$ by $D(\bar{x})=[\overline{f(p)}, \bar{x}]$, which is called an inner derivation on $R / P$. Hence (2) can be written as

$$
[D(\bar{x}), \bar{x}]_{n-1}=0
$$

for all $\bar{x} \in R / P$. Using [6, Theorem], it follows that $[D(\bar{x}), \bar{x}]=0$. Posner's second theorem implies $[\overline{f(p)}, \bar{x}]=0$ for all $x \in R, p \in P$, which means that $\overline{f(p)}$ lies in the centre of $R / P$. In particular, we have $\pi \overline{f(p)}=0$. It follows that the mapping $\bar{f}: R / P \rightarrow A, \bar{f}(\bar{x})=\pi \overline{f(x)}$ is well defined. It is easy to verify that $\bar{f}$ is additive and satisfies $[\bar{f}(\bar{x}), \bar{x}]_{n}=0$ for all $x \in R$. Using Theorem 1.1 of [2], it follows that $[\bar{f}(\bar{x}), \bar{x}]=0$, which in turn implies $[f(x), x] \in P$, as desired. The proof is completed.

Posner's first theorem [12], which states that compositum of two nonzero derivations on a prime ring with characteristic different from two cannot be a derivation, in general cannot be proved for semiprime rings. However, in case we have a semiprime ring, one can prove the following result (Lemma 1.1.9 of [10]): Let $R$ be a 2-torsion free semiprime ring, and let $D, G: R \rightarrow R$ be derivations such that $D^{2}(x)=G(x)$ holds for all $x \in R$. In this case, $D=0$. This result was the motivation for the following result proved by Vukman [15]: Let $R$ be a 2-torsion free semiprime ring, and let $D, G: R \rightarrow R$ be derivations, such that the mapping $x \longmapsto D^{2}(x)+G(x)$ is centralizing on $R$. In this case, $D$ and $G$ are commuting on $R$. The corollary below generalizes Vukman's result we have just mentioned.

\section{COROLLARY 2}

Let $n>1$ be a fixed integer, let $R$ be a $n$ !-torsion free semiprime ring, and let $D, G$ : $R \rightarrow R$ be derivations satisfying the relation $\left[D^{2}(x)+G(x), x\right]_{n}=0$ for all $x \in R$. In this case, $D$ and $G$ map $R$ into $Z(R)$.

Proof. Since all the assumptions of Theorem 1 are fulfilled, one can conclude that the mapping $x \longmapsto D^{2}(x)+G(x)$ is commuting on $R$, whence it follows by Theorem 4 
in [15] that $D$ and $G$ are both commuting on $R$. Since any commuting derivation on a semiprime ring maps the ring into its centre (see, for example, the end of the proof of Theorem 2.1 in [17]), the proof of the corollary is complete.

In case $n=2$, Corollary 2 has been recently proved by Fošner and Vukman in [9].

\section{References}

[1] Baxter W E and Martindale III W S, Jordan homomorphisms of semiprime rings, $J$. Algebra 56 (1979) 457-471

[2] Beidar K I, Fong Y, Lee P-H and Wong T-L, On additive maps of prime rings satisfying the Engel condition, Commun. Algebra 25 (1997) 3889-3902

[3] Beidar K I, Martindale III W S and Mikhalev A V, Rings with generalized identities (1996) (New York: Marcel Dekker, Inc.)

[4] Brešar M, On a generalization of the notion of centralizing mappings, Proc. Amer. Math. Soc. 114 (1992) 641-649

[5] Brešar M, Commuting traces of biadditive mappings, commutativity-preserving mappings and Lie mappings, Trans. Amer. Math. Soc. 335 (1993) 525-546

[6] Brešar M, Applying the theorem on functional identities, Nova J. Math. Game Th. Algebra 4 (1995) 43-54

[7] Brešar M, Functional identities: a survey, Contemp. Math. 259 (1999) 93-103

[8] Brešar M, Chebotar M and Martindale III W S, Functional identities (2007) (Schwitzerland: Birkäuser Verlag)

[9] Fošner A and Vukman J, Some results concerning additive mappings and derivations on semiprime rings, Publ. Math. Debrecen 78 (2011) 575-581

[10] Herstein I N, Rings with involution, Chicago Lectures in Math. (1976) (Chicago London: Univ. of Chicago Press)

[11] Lanski C, An Engel condition with derivation, Proc. Amer. Math. Soc. 118 (1993) 731734

[12] Posner E C, Derivations in prime rings, Proc. Amer. Math. Soc. 8 (1957) 1093-1100

[13] Vukman J, Commuting and centralizing mappings in prime rings, Proc. Amer. Math. Soc. 109 (1990) 47-52

[14] Vukman J, On derivations in prime rings and Banach algebras, Proc. Amer. Math. Soc. 116 (1992) 877-884

[15] Vukman J, Derivations on semiprime rings, Bull. Austral. Math. Soc. 53 (1996) 35335

[16] Vukman J and Kosi-Ulbl, On some equations related to derivations in rings, Internat. $J$. Math. and Math. Soc. 17 (2005) 2703-2710

[17] Vukman J, Identities with derivations on rings and Banach algebras, Glasnik Mat. Vol. 40 (2005) 189-199 